\title{
Targeted inhibition of Sp1-mediated transcription for antiangiogenic therapy of metastatic human gastric cancer in orthotopic nude mouse models
}

\author{
LIWEI WANG ${ }^{1,2,4}$, XIAOHONG GUAN ${ }^{1}$, JUN ZHANG ${ }^{3,4}$, ZHILIANG JIA ${ }^{4}$, \\ DAOYAN WEI ${ }^{4}$, QIANG LI ${ }^{4}$, JAMES YAO ${ }^{4}$ and KEPING XIE ${ }^{4,5}$
}

\begin{abstract}
${ }^{1}$ The Key Laboratory of Antibody Technology of State Ministry of Health, Nanjing Medical University, Nanjing 210029; ${ }^{2}$ Cancer Center, Jiaotong University Affiliated Shanghai First People's Hospital, Shanghai 200080; ${ }^{3}$ Department of Surgery, Jiaotong University Affiliated Ruijin Hospital, Shanghai 200025, P.R. China; Departments of ${ }^{4}$ Gastrointestinal Medical Oncology and ${ }^{5}$ Cancer Biology, The University of Texas M.D. Anderson Cancer Center, Houston, TX 77030, USA
\end{abstract}

Received October 26, 2007; Accepted December 28, 2007

\begin{abstract}
Overexpression of the transcription factor $\mathrm{Sp} 1$ may play a critical role in human gastric cancer angiogenesis. In the present studies, we determined whether targeting Sp1 has a therapeutic benefit. Treatment with mithramycin A (MIT) suppressed the expression of Sp1 and its downstream target genes in both human gastric cancer cell culture and tumors growing in nude mice. The molecular responses were accompanied by a significant inhibition of gastric cancer angiogenesis, growth and metastasis. Conversely, treatment with bevacizumab (BVZ), a neutralizing antibody against VEGF A, suppressed human gastric cancer growth in nude mice in a dose-dependent manner. Gene expression analyses revealed that treatment with low dose of BVZ substantially upregulated the expression of Sp1 and its downstream target genes, including VEGF and EGFR, in tumor tissues, whereas it did not have this effect on gastric cancer cells in culture. Combined treatment with BVZ and MIT produced synergistic tumor suppression, which was consistent with suppression of the expression of $\mathrm{Sp} 1$ and its downstream target genes. Thus, treatment with BVZ may block VEGF function but activate the pathway of its expression via positive feedback. Collectively, $\mathrm{Sp} 1$ is an important regulator of the expression
\end{abstract}

Correspondence to: Dr Keping Xie, Department of Gastrointestinal Medical Oncology, Unit 426, The University of Texas M.D. Anderson Cancer Center, 1515 Holcombe Boulevard, Houston, TX 77030, USA

E-mail: kepxie@mail.mdanderson.org

Dr Xiaohong Guan, The Key Laboratory of Antibody Technology of State Ministry of Health, Nanjing Medical University, Nanjing 210029, P.R. China

E-mail:xhguan@163.com

Key words: angiogenesis, metastasis, mithramycin A, Sp1, VEGF of multiple angiogenic factors and functional status of Sp1 signaling pathway may profoundly affect the angiogenic phenotype of and effectiveness of antiangiogenic strategies for human gastric cancer.

\section{Introduction}

Although the incidence of gastric cancer declined in the West from the 1940s to the 1980s, it remains a major public health problem throughout the world (1). In Asia and parts of South America in particular, it is the most common epithelial malignancy and leading cause of cancer-related deaths. In fact, gastric cancer remains the second most frequently diagnosed malignancy worldwide and causes $12 \%$ of all cancer-related deaths each year $(1,2)$. A full understanding of the cellular and molecular mechanisms of the development and progression of gastric cancer is crucial for identifying new targets of effective treatment modalities for this deadly disease.

The aggressive nature of human metastatic gastric carcinoma is attributed to mutations of various oncogenes and tumor suppressor genes (3-6) and abnormalities in various growth factors and their receptors $(4,5)$. These abnormalities affect the downstream signal transduction pathways involved in the control of various aspects of cancer biology, including angiogenesis (4-6). Specifically, these perturbations may confer an elevated angiogenic phenotype to gastric cancer cells. The status of angiogenesis as assessed according to the MVD is usually correlated with the expression of a variety of angiogenic factors, including bFGF, TGF, MMP-2, EGFR, IGFR, IL-8 and VEGF (7-11). Early studies showed that MVD and various angiogenic factors are related to tumor development and progression, including prediction of patient survival (12-22).

The molecular mechanisms behind abnormal expression of multiple molecules and its relationship with MVD are not entirely clear. Our recent studies of human pancreatic and gastric cancer have linked abnormal $\mathrm{Sp} 1$ activation to overexpression of multiple Sp1 downstream genes $(23,24)$. These 
findings led us to propose that abnormal activation of Sp1 may cause overexpression of multiple angiogenic molecules, which in turn render tumor cells highly angiogenic and aggressive $(23,24)$. However, it is unclear whether and, if so, how treatments targeting Sp1 impact gastric cancer angiogenesis and whether and, if so, how antiangiogenesis therapies impact the expression of Sp1 and its downstream target genes, including VEGF.

$\mathrm{Sp} 1$ is a zinc finger transcription factor that is important to the transcription of many cellular and viral genes that contain GC boxes in their promoters. Researchers have cloned additional transcription factors similar to $\mathrm{Sp} 1$ in their structural and transcriptional properties Sp2, Sp3 and Sp47, thus forming the Sp1 multigene family (25). Although Sp1 has been perceived to be a basal transcription factor since its discovery, increasing evidence suggests that it regulates a variety of biological functions, including cell survival, growth and differentiation and tumor development and progression (25-29). We previously reported that $\mathrm{Sp} 1$ overexpression is directly correlated with the angiogenic potential of and poor prognosis for human gastric and pancreatic cancer $(23,24)$. Therefore, Sp1 inhibitors such as mithramycin A (MIT) may have profound antiangiogenic effects.

MIT is an aureolic acid-type polyketide produced by various soil bacteria of the genus Streptomyces $(30,31)$. In the past, MIT has been used to manage hypercalcemia in patients with bone metastases from various malignancies, while some have also used it to treat Paget's disease and several types of cancer, including testicular carcinoma, chronic myeloid leukemia and acute myeloid leukemia $(32,33)$. Furthermore, researchers have shown that MIT acts as a neuroprotective drug (34). MIT binds to GC-rich regions in chromatin and interferes with the transcription of genes that bear GC-rich motifs in their promoters $(32,35)$. Its mechanism of action involves a reversible interaction with double-stranded DNA with GC-base specificity. MIT is believed to act, in part, by selectively regulating transcription of genes that have GC-rich promoter sequences (36). In addition, recent studies have shown that MIT sensitizes tumor cells to apoptosis induced by tumor necrosis factor and inhibits p53-mediated transcriptional responses $(37,38)$. Inhibition of Sp1 activity is considered to be a major mechanism of the antitumor activity of MIT. Thus, we performed the present study to determine whether Sp1 is an effective target for antiangiogenic therapy of gastric cancer.

\section{Materials and methods}

Chemicals and reagents. MIT (1 mg/vial crystal powder; lot 055K4011) was purchased from Sigma Chemical Co. (St. Louis, MO) and diluted in sterile water. BVZ $(25 \mathrm{mg} / \mathrm{ml}$; NDC 50242-060-01) was purchased from Genentech, Inc. (South San Francisco, CA). For animal experiments, MIT (0.1-0.8 mg/kg body weight) and BVZ (25-100 $\mu \mathrm{g} / \mathrm{mouse})$ were administered by intraperitoneal injection twice a week or as indicated otherwise.

Cell lines and culture conditions. The N87 human gastric cancer cell line was purchased from the American Type
Culture Collection (Manassas, VA) and the SK-GT5 human gastric cancer cell line was obtained from Dr Gary K. Schwartz (Memorial Sloan-Kettering Cancer Center). The cell lines were maintained in plastic flasks as adherent monolayers in minimal essential medium supplemented with $10 \%$ fetal bovine serum, sodium pyruvate, non-essential amino acids, L-glutamine and a vitamin solution (Flow Laboratories, Rockville, MD).

Animals. Female athymic nude mice were purchased from The Jackson Laboratory (Bar Harbor, ME). The mice were housed in laminar flow cabinets under specific pathogenfree conditions and used when they were 8-week old. The animals were maintained in facilities approved by the Association for Assessment and Accreditation of Laboratory Animal Care in accordance with the current regulations and standards of the US Department of Agriculture, US Department of Health and Human Services and the National Institutes of Health.

Western blot analysis. Whole-cell lysates were prepared from human gastric cancer cell cultures. Standard Western blotting was performed using polyclonal rabbit antibodies against human and mouse Sp1, VEGF and EGFR (Santa Cruz Biotechnology, Santa Cruz, CA) and the anti-rabbit IgG antibody, a horseradish peroxidase-linked $\mathrm{F}\left(\mathrm{ab}^{\prime}\right)_{2}$ fragment obtained from a donkey (Amersham, Arlington Heights, IL). Equal protein-sample loading was monitored by probing the same membrane filter with antibodies against anti- 3 -actin (23). The probe proteins were detected using the Amersham enhanced chemiluminescence system according to the manufacturer's instructions.

Animal models of tumor growth and metastasis. To prepare tumor cells for inoculation, cells in the exponential growth phase were harvested by brief exposure to a $0.25 \%$ trypsin/ $0.02 \%$ ethylenediaminetetraacetic acid solution (wt/vol). Cell viability was determined by using trypan blue exclusion, and only single-cell suspensions that were $>95 \%$ viable were used. Tumor cells $\left(1 \times 10^{6}\right.$ cells/mouse $)$ were then injected into the subcutis or the wall of stomach of nude mice in groups of 10 . The animals were sacrificed 60 days after the tumor-cell injection or when they had become moribund. Next, the primary gastric tumors were harvested and weighed. In addition, the liver of each mouse and regional lymph nodes were fixed in Bouin's solution for $24 \mathrm{~h}$ to differentiate the neoplastic lesions from the organ parenchyma; metastases on the surface of the liver were counted (double-blinded) with a dissecting microscope and nodal metastasis was determined by histopathology.

Immunohistochemistry of human tumor xenograft specimens. For VEGF and Sp1 staining, sections (5 $\mu \mathrm{m}$ thick) of formalin-fixed, paraffin-embedded tumor specimens were deparaffinized in xylene and rehydrated in graded alcohol. For CD31 staining, frozen sections (6 $\mu \mathrm{m}$ thick) were fixed with acetone. Endogenous peroxidase was blocked using $3 \%$ hydrogen peroxide in PBS for $12 \mathrm{~min}$. The sections were incubated for $20 \mathrm{~min}$ at room temperature with a proteinblocking solution consisting of PBS (pH 7.5) containing 5\% 
normal horse serum and $1 \%$ normal goat serum and then incubated overnight at $4^{\circ} \mathrm{C}$ in anti-CD31, anti-Sp1, or antiVEGF antibodies. Sp1 and VEGF expression and MVD status were assessed as described above $(24,39)$.

Chromatin immunoprecipitation. Chromatin was prepared from cells and tumors as described previously (40). Chromatin immunoprecipitation (ChIP) assay was performed using the chromatin immunoprecipitation assay kit (Upstate Cell Signaling Solutions, Lake Placid, NY) according to the manufacturer's instructions. Briefly, DNA cross-binding proteins were cross-linked with DNA and lysed in sodium dodecyl sulfate lysis buffer. The lysate was sonicated to shear DNA to 200-500 bp. After preclearing with a salmon sperm DNA/protein A agarose-50\% slurry for 30 min at $4^{\circ} \mathrm{C}$, chromatin samples were immunoprecipitated overnight with no antibody or an anti-Sp1 antibody (PEP2). The region between -224 and -53 bp of the $\mathrm{Sp} 1$ promoter was amplified using the following primers: sense, 5'-caggcacgcaacttagtc-3', and antisense, 5'-gtaaggaggagggagcag-3'. Polymerase chain reaction products were separated on a $1.5 \%$ agarose gel, stained with ethidium bromide, and visualized under ultraviolet light.

Statistical analysis. For in vitro and in vivo experimental studies, each experiment was performed independently at least twice with similar results; one representative experiment is presented. The significance of the in vitro data was determined using Student's t-test (2-tailed), whereas the significance of the in vivo data was determined using the 2-tailed Mann-Whitney U test. For the in vivo experiments, overall animal survival was calculated by the method of Kaplan and Meier. Log-rank test was used to compare the survival duration between groups. $\mathrm{P} \leq 0.05$ was deemed significant.

\section{Results}

MIT inhibited Spl expression and the expression of its downstream target molecules in human gastric cancer cells. N87 and GT5 cells were incubated in medium or medium containing different concentrations of MIT for $24 \mathrm{~h}$. MIT produced dose-dependent growth inhibition with $\mathrm{IC}_{50} 1.24 \mu \mathrm{M}$ for GT5 cells and $1.13 \mu \mathrm{M}$ for N87 cells (Fig. 1A). Protein extracts were used for Western blot analyses of expression of Sp1 and its downstream molecules. As shown in Fig. 1B, MIT produced a concentration-dependent inhibition of Sp1 and VEGF protein expression. To further determine whether MIT compete with Sp1 for Sp1-binding sites, we performed the ChIP assay. As shown in Fig. 1C, MIT inhibited Sp1 binding to the Sp1-binding sites of its own promoter. Our studies have demonstrated that MIT inhibits Sp1 expression and its downstream molecule VEGF.

MIT inhibited angiogenesis of human gastric cancer in animal models. N87 and GT5 cells were either injected into the subcutis (ectopic model) or the wall of stomach (orthotopic model) of mouse. MIT treatment started when tumor sizes reached around 3-4 $\mathrm{mm}$ in diameter. MIT treatment produced dose-dependent inhibition of tumor growth in both
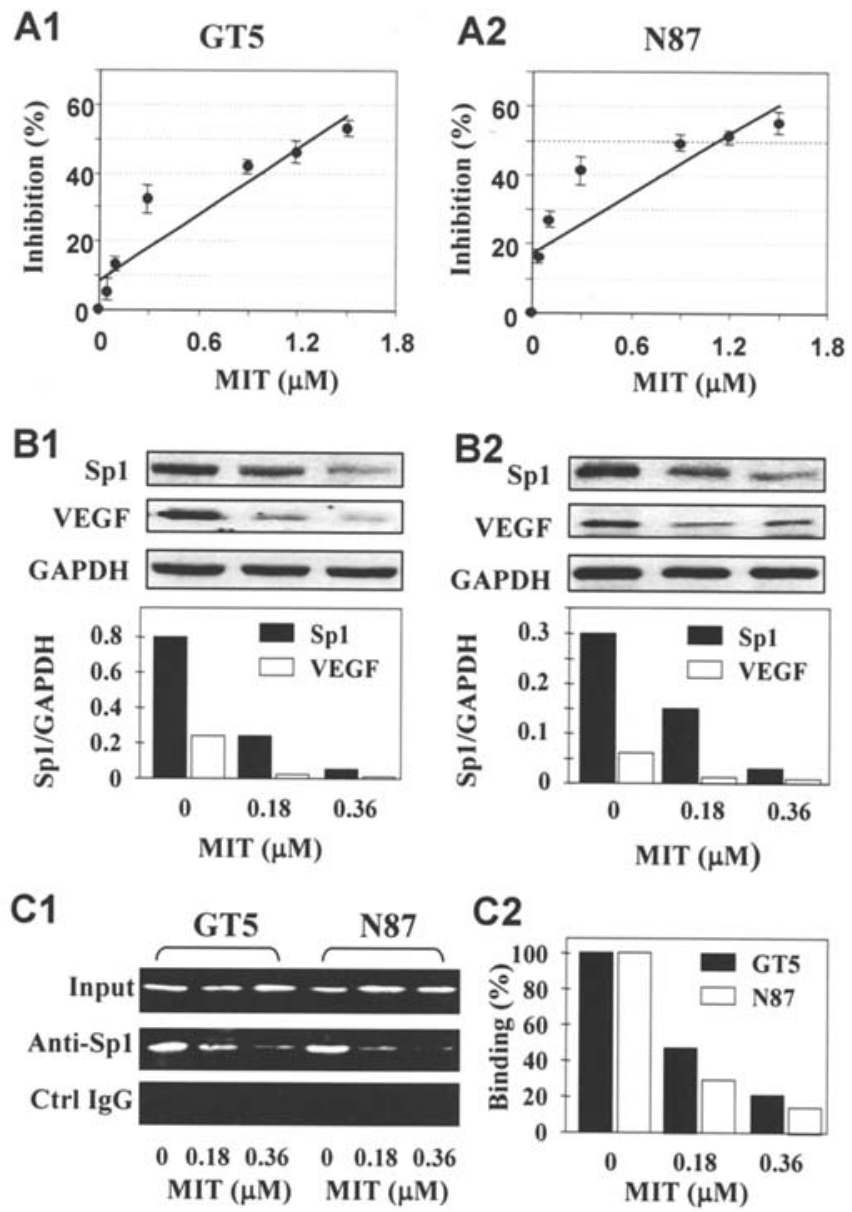

Figure 1. Inhibition of expression of Sp1 and its downstream molecules by MIT in human gastric cancer cells. N87 and GT5 cells were incubated in medium or medium containing 0-1.5 $\mu \mathrm{M}$ MIT for $24 \mathrm{~h}$. (A) Growth inhibition was determined by MTT assay. (B) Total protein lysates were extracted for Western blot analyses of expression of Sp1 and VEGF. (C) Chromatin was prepared from the cells and chromatin immunoprecipitation (ChIP) assay was performed using the chromatin immunoprecipitation assay kit as described in Materials and methods. Note that MIT inhibits Sp1 expression and its downstream molecules. This was one representative experiment of two with similar results.

ectopic (Fig. 2A) and orthotopic models (Fig. 2B). Moreover, the incidence of liver metastasis in control mice was $80 \%$ (8/10 mice), whereas no liver metastasis was detected in mice receiving MIT treatment (from 0.2 to $0.8 \mathrm{mg} / \mathrm{kg}$ ), suggesting a total inhibition of metastasis by MIT treatment. The residual tumors were harvested and angiogenesis was assessed by CD31 staining. As shown in Fig. 2C, MIT treatment substantially reduced MVD. Moreover, Western blot analyses showed that MIT treatment inhibited the expression of VEGF and EGFR, which was consistent with significant inhibition of Sp1 expression (Fig. 2D). No discernible inhibition of Sp1 expression was observed in normal tissues including liver, stomach and intestine (data not shown), suggesting that MIT selectively inhibited $\mathrm{Sp} 1$ expression in tumor cells growing in animals. These data suggested that inhibition of Sp1 expression by MIT exhibited a marked antiangiogenesis and antitumor activity.

$B V Z$ upregulated Spl expression in human gastric cancer cells in animal models. To determine whether antiangio- 

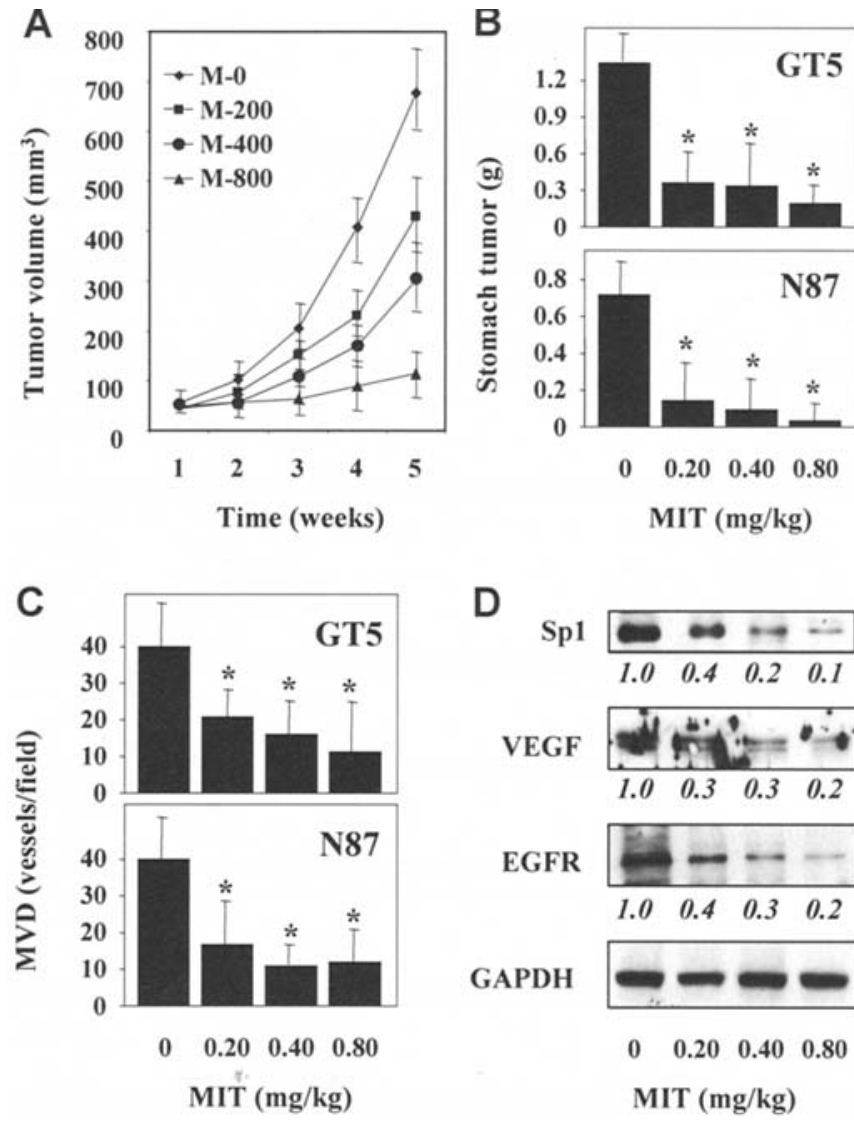

Figure 2. Inhibition of human gastric cancer angiogenesis by MIT in animal models. (A) GT5 cells were injected into the subcutis of nude mice $(n=5)$. When tumors reached around $4 \mathrm{~mm}$ in diameter, the animals received different doses of MIT [0.20 mg/kg (M-200), $0.40 \mathrm{mg} / \mathrm{kg}$ (M-400), and $0.80 \mathrm{mg} / \mathrm{kg}(\mathrm{M}-800)$ ] via intraperitoneal injection twice a week. Tumors were measured once every week, and at each measurement, the mean \pm standard deviation (SD) tumor volume in the five mice in each group was calculated. (B) N87 and GT5 cells were injected into the wall of stomach of nude mice $(n=5)$. When tumors reached around $4 \mathrm{~mm}$ in diameter (around 15 days after tumor injection), the animals received different doses of MIT $(0.200 .40$ and $0.80 \mathrm{mg} / \mathrm{kg})$ via subcutaneous injection twice a week. Primary gastric tumors in the control mice and mice that received MIT were weighed at the time of experiment termination, and the weights were expressed as the mean \pm SD. (C) The residual tumors were harvested and CD31 staining was performed using frozen sections and MVD was assessed by counting. (D) Total protein lysates were prepared from tumor tissues of mice that received PBS or MIT treatment. Western blot was performed to determine the expression of Sp1 and VEGF and EGFR. The asterisks indicate statistical significance $(\mathrm{P}<0.01)$ in a comparison between the treated and respective control groups. This was one representative experiment of two with similar results.

genesis therapies including those targeting VEGF signaling impact Sp1 pathway, we treated both N87 and GT5 tumor around 3-4 $\mathrm{mm}$ in diameter with different doses of BVZ. As shown in Fig. 3, at a high dose BVZ inhibited tumor growth (Fig. 3A1) and Sp1 expression accordingly (Fig. 3A2); whereas low dose of BVZ did not significantly inhibit tumor growth (Fig. 3A1) but slightly upregulated Sp1 expression (Fig. 3A2). Next, we started the BVZ treatment when tumors reached 6-7 $\mathrm{mm}$ in diameter with same doses of BVZ. As shown in Fig. 3B, both low and high doses of BVZ were ineffective in inhibiting tumor growth (Fig. 3B1) but significant increase in Sp1 expression was observed (Fig. 3B2). The altered expression of $\mathrm{Sp} 1$ protein directly correlated with the alterations of VEGF, and EGFR expression in
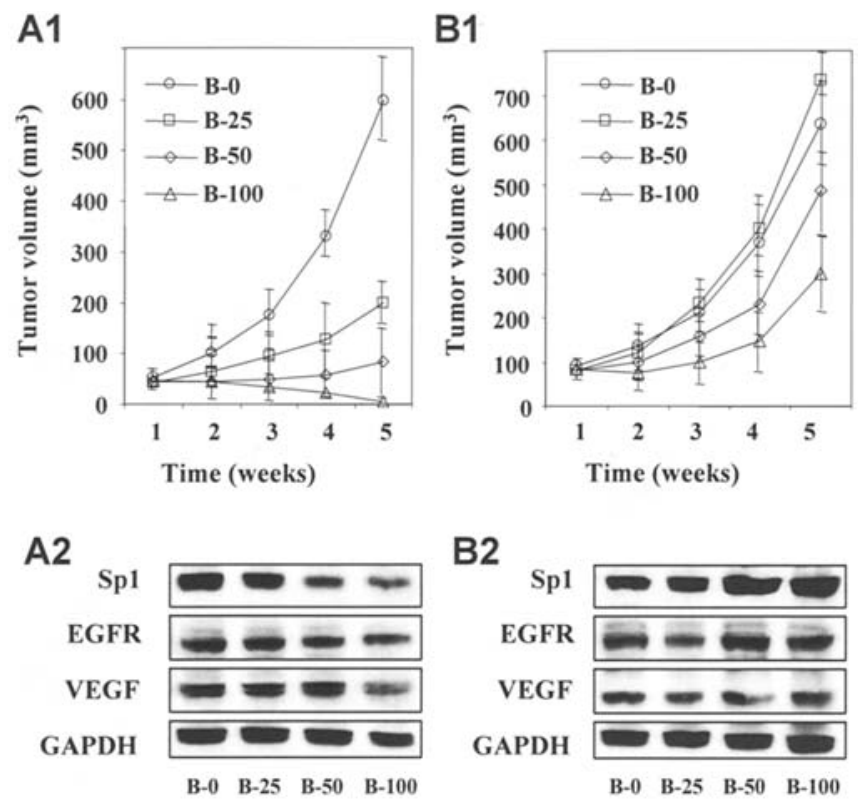

Figure 3. Upregulation of Sp1 expression in human gastric cancer by BVZ in animal models N87 and GT5 tumor cells were injected into the subcutis of nude mice $(n=5)$. BVZ treatments $[0.025 \mathrm{mg}(\mathrm{B}-25), 0.050 \mathrm{mg}$ (B-50) and $0.100 \mathrm{mg}$ (B-100) via intraperitoneal injection twice a week] started when tumors reached around 3-4 mm in diameters (A) or around 6-7 mm in diameter (B). Tumors were measured once every week, and at each measurement, the mean \pm standard deviation (SD) tumor volume in the five mice in each group was calculated (A1 and B1). Tumor tissues were collected for analysis of expression of Sp1, VEGF, and EGFR by Western blotting (A2 and B2). This was one representative experiment of two with similar results.

tumors growing in mice which received BVZ treatment as compared to those that received control treatments (Fig. 3A2 and B2). These data suggested that ineffective dose of BVZ (BVZ-resistant tumors) upregulated the expression of $\mathrm{Sp} 1$ and its down-stream molecules.

Effects of treatment with BVZ and MIT on the growth and gene expression in human gastric cancer cells. SK-GT5 and N87 cells incubated were in a medium alone or a medium containing 25, 50 and $100 \mu \mathrm{g} / \mathrm{ml} \mathrm{BVZ} \mathrm{for} \mathrm{24-72} \mathrm{h.} \mathrm{BVZ}$ did not affect the growth of SK-GT5 cells (Fig. 4A1) or N87 cells (Fig. 4A2) in vitro. As shown in Fig. 4B, neutralization of VEGF did not affect the expression of Sp1 or its major downstream molecule, VEGF. This result was consistent with previous findings showing that BVZ primarily blocks the autocrine effect of VEGF on tumor angiogenesis (43-47). In contrast, treatment with MIT produced dose-dependent cytotoxic effects in both SK-GT5 cells and N87 cells (Fig. 4A1 and A2) and effectively inhibited the expression of Sp1 and its downstream molecule VEGF (Fig. 4B). These data suggest that BVZ does not have a direct effect on tumor cells or on the expression of Sp1 and its downstream molecules, but that MIT does.

Synergistic antitumor effects of BVZ and MIT in human gastric cancer model. To determine whether modulating $\mathrm{Sp} 1$ expression and/or activity impact the efficacy of antiangiogenic treatment of BVZ, we injected SK-GT5 cells subcutaneously into nude mice. When their tumors reached $4 \mathrm{~mm}$ 


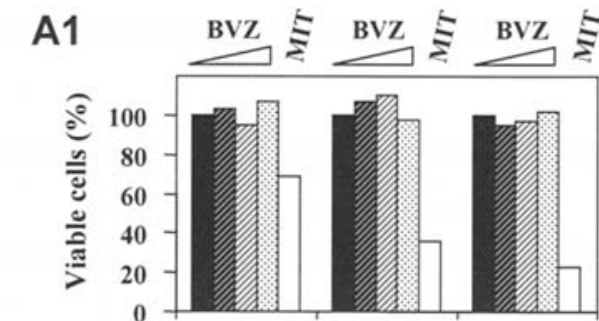

A2
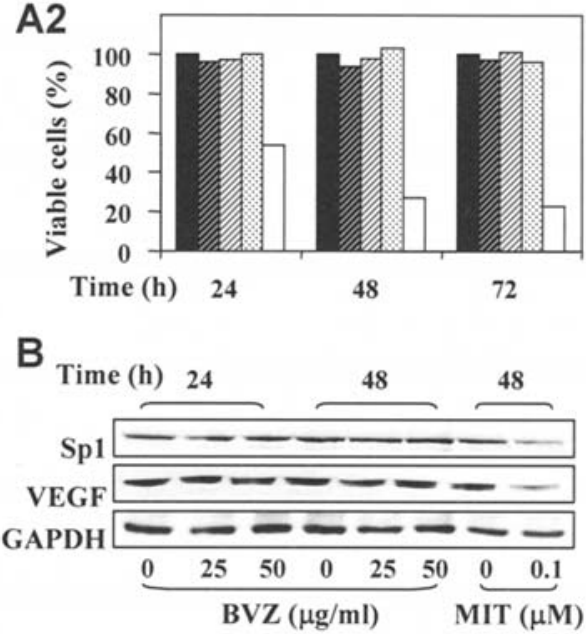

Figure 4. Effects of treatment with BVZ and MIT on the growth of and gene expression in human gastric cancer cells in vitro. (A) GT5 (A1) and N87 (A2) cells were incubated for 1-3 days in a medium alone or a medium containing 25, 50 and $100 \mu \mathrm{g} / \mathrm{ml} \mathrm{BVZ}$. The viable cells were determined every $24 \mathrm{~h}$ by MTT assay. As controls, GT5 (A1) and N87 (A2) cells were treated with $0.9 \mu \mathrm{M}$ MIT. (B) GT5 cells were incubated for 24 or $48 \mathrm{~h}$ in a

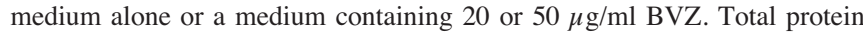
lysates were harvested from the cell cultures, and the level of Sp1, VEGF protein expression was determined using Western blot analysis. Equal protein-sample loading was monitored by probing the same membrane filter with an anti-GAPDH. As controls, GT5 cells were treated for $48 \mathrm{~h}$ with $0.1 \mu \mathrm{M}$ MIT. This was one representative experiment of two with similar results.

in diameter, we gave the animals BVZ $(25 \mu \mathrm{g} / \mathrm{mouse})$ alone or in combination with MIT $(0.1 \mathrm{mg} / \mathrm{kg}$, with slight antitumor effects) via intraperitoneal injection twice a week. Administration of BVZ or MIT alone produced marginal antitumor activity whereas combined use of BVZ at a dose of $25 \mu \mathrm{g}$ and MIT at a dose of $0.10 \mathrm{mg} / \mathrm{kg}$ produced a synergistic antitumor activity (Fig. 5A). We observed no discernable effect on body weight in the mice that received BVZ alone (Fig. 5B). However, at the highest dose given $(0.4 \mathrm{mg} / \mathrm{kg})$, MIT slightly reduced body weight (data not shown). Additionally, the mice that received combination of BVZ and MIT had body weights similar to those of the control mice that received PBS (data not shown). Therefore, the combined use of BVZ and MIT resulted in higher antitumor activity than the use of BVZ or MIT alone did without an increase in toxicity, suggesting that the combination treatment has a significant therapeutic benefit. Furthermore, we injected SK-GT5 cells into the stomach of nude mice and then administered treatment to them as described in Fig. 2. We monitored animal survival daily until termination of the experiment 120 days after tumor cell injection. We found that the mice that received BVZ or MIT alone had a slightly increased survival rate $(93 \pm 12$ days) when compared with those that received PBS only $(63 \pm 12$ days $)$, whereas the mice
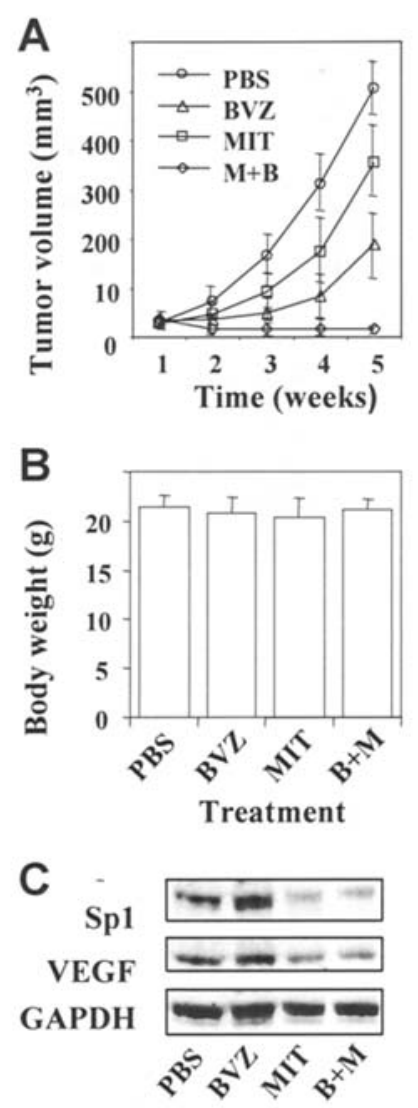

Treatment

Figure 5. Synergistic antitumor effects of BVZ and MIT in human gastric cancer model. GT5 cells were injected subcutaneously into nude mice. When their tumors reached $4 \mathrm{~mm}$ in diameter, we gave the animals different doses of BVZ (25 $\mu \mathrm{g} /$ mouse $)$ alone or in combination with MIT $(0.1 \mathrm{mg} / \mathrm{kg})$ via intraperitoneal injection twice a week. (A) Tumors were measured once every week, and at each measurement, the mean \pm standard deviation (SD) tumor volume in the five mice in each group was calculated. (B) Mouse body weights were measured. (C) Total protein lysates were harvested from tumor tissues, and the expression levels of Sp1, and VEGF protein were determined using Western blot analyses. Equal protein-sample loading was monitored by probing the same membrane filter with an anti-GAPDH antibody. This was one representative experiment of two with similar results.

that received BVZ and MIT had significantly longer survival (>120 days). Furthermore, the incidence of tumor growth in the stomach and of metastasis in the liver and/or other organs was significantly lower in the mice that received BVZ and MIT.

Upregulation of Sp1 and VEGF expression by treatment with $B V Z$ and its reversal by treatment with MIT. To determine the molecular basis for the synergistic effect of treatment with BVZ and MIT, we performed Western blot analysis using total protein lysates extracted from the SK-GT5 tumor tissue specimens collected from mice that received treatment with PBS, BVZ, MIT, or B+M. As shown in Fig. 5C, treatment with BVZ alone increased expression of Sp1 and its downstream molecule VEGF. However, treatment with MIT alone suppressed Sp1 and VEGF expression, which was consistent with reduced MVD, whereas treatment with BVZ at low dose alone did not significantly reduce MVD, which was consistent with increased $\mathrm{Sp} 1$ expression (data 
not shown). These data suggested that neutralization of VEGF function by BVZ may upregulate the expression of Sp1 via a positive feedback loop and lead to increased VEGF expression.

\section{Discussion}

In this study, we found that MIT treatment suppressed the expression of $\mathrm{Sp} 1$ and its downstream target genes in both gastric cancer cell culture and tumors growing in nude mice. The molecular responses were accompanied by a significant inhibition of gastric cancer angiogenesis, growth and metastasis with selective inhibition of Sp1 in tumor cells not in normal tissues, which was consistent with no discernible systemic side-effect. Interestingly, BVZ treatment upregulated the expression of $\mathrm{Sp} 1$ and its downstream target genes, including VEGF and EGFR, in tumors. Combined treatment with BVZ and MIT produced synergistic tumor suppression, which was consistent with suppression of Sp1 upregulation. Given the fact that $\mathrm{Sp} 1$ is an important regulator of the expression of multiple angiogenic factors, BVZ-initiated upregulation of $\mathrm{Sp} 1$ and subsequent overexpression of its downstream target genes may profoundly affect the potential angiogenic phenotype and effectiveness of antiangiogenic strategies for human gastric cancer. Therefore, our study further demonstrated the crucial role of Sp1 signaling in tumor angiogenesis.

Angiogenesis is essential for tumor growth and metastasis (38). Numerous lines of evidence have shown that angiogenesis plays significant clinicopathological roles in patients with several types of tumors (39-45). Many strategies have been developed to inhibit angiogenesis for cancer therapy. Most strategies are directed at individual angiogenic factors. However, many tumors exhibit resistance and/or acquire resistance and the underlying mechanisms remain unclear. For example, while BVZ has demonstrated significant antitumor activity in a wide variety of xenograft models, clinical activity for BVZ when used as single agent has limited in human studies. Like other VEGF inhibitor, BVZ-based antiangiogenic approaches are generally developed in combination and found to be more effective when combined with chemotherapy and radiation therapy (44). Various mechanisms for synergy between antiangiogenic therapy and chemotherapy and radiation therapy have been proposed. For instance, therapy targeting VEGF may normalize tumor vasculature, decrease interstital fluid pressure, and enhance chemotherapy delivery $(45,46)$. Alternatively, others have proposed that neuropilin receptors may mediate resistance to apoptosis which can be reversed by agents such as BVZ (47). In our present study, we provide evidence that $\mathrm{Sp} 1$ signaling and function impact antiangiogenesis therapies. Our finding may represent a novel mechanism for the limited effectiveness of antiangiogenic therapies that target individual molecules individually, highlighting the importance of combined strategies for antiangiogenesis therapy.

It is realized that gastric cancer overexpresses a variety of angiogenic factors, including bFGF, TGF, MMP-2, EGFR, IGFR, IL-8, and VEGF (7-11) and simultaneously interfering with multiple target molecules is clinically challenging. Understanding the underlying mechanism for overexpression of various proangiogenic factors would help design effective strategies targeted those factors $(6,9)$. Previous studies have shown that overexpression of Sp1 is correlated with MVD in human gastric cancer tissue and that manipulating the Sp1 expression level using a small interfering RNA approach significantly inhibits the tumor angiogenic phenotype $(6,9)$. This antiangiogenic effect of knocking down Sp1 expression is consistent with reduced expression of several signaling molecules in the signaling pathways that play important roles in the regulation of tumor angiogenesis $(24,28,29)$. In fact, one of our recent studies showed that inhibition of VEGF expression and the resulting antitumor effect of celecoxib are mediated at least in part by suppression of Sp1 activity (24). Collectively these lines of evidence indicate that $\mathrm{Sp} 1$ plays an important role in regulation of angiogenesis in human gastric cancer. Furthermore, as shown in the present study, blockade of Sp1 activity by MIT suppresses tumor angiogenesis via downregulating its downstream target genes, including VEGF and EGFR. Therefore, we offered direct experimental evidence of $\mathrm{Sp} 1$ as effective target for antiangiogenic therapy of human gastric cancer.

Finally, we offered experimental evidence that antiangiogenic therapies impact Sp1 signaling and influence the efficacy of those therapies, while the potential mechanisms underlying Sp1 upregulation by BVZ treatment is unclear. Further studies are clearly necessary to address certain questions. First, is the perturbation of Sp1 signaling and function a general response to all antiangiogenesis strategies targeting individual angiogenesis factors, particularly those that are known to be under Sp1 regulation? Second, are there other general signaling pathways altered during antiangiogenic therapies? Third, how can Sp1 be effectively inhibited by targeting? What are the molecular basis for differential inhibitions of Sp1 expression by MIT in tumor tissues and normal tissues? Further addressing these questions and our current findings will have significant implications in better understanding gastric cancer pathogenesis and designing effective targeted therapies. Specifically, our findings not only provide researchers with a novel paradigm of synergism between antiangiogenic agents and inhibitors of transcription factors such as Sp1, but also will help clinicians design combination drug therapies for improved anti-tumor activity. Collectively, our study suggests that blockade of Sp1 expression and function may be a promising approach to treatment of human gastric cancer.

\section{Acknowledgments}

We thank Gerard Quinones for expert help in the preparation of the manuscript and Don Norwood for editorial comments. The study was supported with a Grant 054119628 from the Natural Science Foundation of Shanghai City Government, China (to L.W.); and a Research Scholar Grant CSM106640 from the American Cancer Society and a grant 1R01CA093829 from the National Cancer Institute, National Institutes of Health (to K.X.).

\section{References}

1. Parkin DM, Bray F, Ferlay J, et al: Global cancer statistics. CA Cancer J Clin 55: 74-108, 2005. 
2. Parkin DM, Pisani P and Ferlay J: Estimates of the worldwide incidence of 25 major cancers. Int J Cancer 80: 827-841, 1999.

3. Zheng L, Wang L, Ajani J, et al: Molecular basis of gastric cancer development and progression. Gastric Cancer 7: 61-77, 2004.

4. Chan AO, Luk JM and Hui WM: Molecular biology of gastric carcinoma: from laboratory to bedside. J Gastroenterol Hepatol 14: 1150-1160, 1999.

5. Xie K, Wei D and Huang S: Transcriptional anti-angiogenesis therapy of human pancreatic cancer. Cytokine Growth Factor Rev 17: 147-156, 2006.

6. Siewert JR, Bottcher K, Stein HJ, et al: Relevant prognostic factors in gastric cancer: ten-year results of the German Gastric Cancer Study. Ann Surg 228: 449-461, 1998.

7. Xie K: Interleukin-8 and human cancer biology. Cytokine Growth Factor Rev 12: 375-391, 2001.

8. Xie K, Wei D, Shi Q, et al: Constitutive and inducible expression and regulation of vascular endothelial growth factor. Cytokine Growth Factor Rev 15: 297-324, 2004.

9. Guo YS, Beauchamp, RD, Jin GF, et al: Insulinlike growth factor-binding protein modulates the growth response to insulinlike growth factor 1 by human gastric cancer cells. Gastroenterology 104: 1595-1604, 1993.

10. Yoshikawa T, Tsuburaya A, Kobayashi O, et al: Plasma concentrations of VEGF and bFGF in patients with gastric carcinoma. Cancer Lett 153: 7-12, 2000.

11. Sier CF, Kubben FJ, Ganesh S, et al: Tissue levels of matrix metalloproteinases MMP-2 and MMP-9 are related to the overall survival of patients with gastric carcinoma. Br J Cancer 74: 413-417, 1996.

12. Lu M, Jiang $Y$ and Wang R: The relationship of vascular endothelial growth factor and angiogenesis to the progression of gastric carcinoma. Zhonghua Bing Li Xue Za Zhi 27: 278-281, 1998.

13. Giatromanolaki A, Koukourakis MI, Stathopoulos GP, et al: Angiogenic interactions of vascular endothelial growth factor, of thymidine phosphorylase, and of p53 protein expression in locally advanced gastric cancer. Oncol Res 12: 33-41, 2000.

14. Takahashi R, Tanaka S, Kitadai Y, et al: Expression of vascular endothelial growth factor and angiogenesis in gastrointestinal stromal tumor of the stomach. Oncology 64: 266-274, 2003.

15. Du JR, Jiang Y, Zhang YM, et al: Vascular endothelial growth factor and microvascular density in esophageal and gastric carcinomas. World J Gastroenterol 9: 1604-1606, 2003.

16. La Rosa S, Uccella S, Finzi G, et al: Localization of vascular endothelial growth factor and its receptors in digestive endocrine tumors: correlation with microvessel density and clinicopathologic features. Hum Pathol 34: 18-27, 2003.

17. Kaneko T, Konno H, Baba M, et al: Urokinase-type plasminogen activator expression correlates with tumor angiogenesis and poor outcome in gastric cancer. Cancer Sci 94: 43-49, 2003.

18. Sanz-Ortega J, Steinberg SM, Moro E, et al: Comparative study of tumor angiogenesis and immunohistochemistry for p53, c-ErbB2, c-myc and EGFr as prognostic factors in gastric cancer. Histol Histopathol 15: 455-462, 2000.

19. Tenderenda M, Rutkowski P, Jesionek-Kupnicka D, et al: Expression of CD34 in gastric cancer and its correlation with histology, stage, proliferation activity, p53 expression and apoptotic index. Pathol Oncol Res 7: 129-134, 2001.

20. Chen CN, Cheng YM, Lin MT, et al: Association of color Doppler vascularity index and microvessel density with survival in patients with gastric cancer. Ann Surg 235: 512-518, 2002.

21. Hironaka S, Hasebe $\mathrm{T}$, Kamijo $\mathrm{T}$, et al: Biopsy specimen microvessel density is a useful prognostic marker in patients with $\mathrm{T}(2-4) \mathrm{M}(0)$ esophageal cancer treated with chemoradiotherapy. Clin Cancer Res 8: 124-130, 2002.

22. Kim HS, Kang HS, Messam CA, et al: Comparative evaluation of angiogenesis in gastric adenocarcinoma by nestin and CD34. Appl Immunohistochem Mol Morphol 10: 121-127, 2002.

23. Shi Q, Le X, Peng Z, et al: Constitutive Sp1 activity is essential for differential constitutive expression of vascular endothelial growth factor in human pancreatic adenocarcinoma. Cancer Res 61: 4143-4154, 2001.

24. Wang L, Wei D, Le X, et al: Transcription factor Sp1 expression is a significant predictor of survival in human gastric cancer. Clin Cancer Res 9: 6371-6380, 2003.

25. Black AR, Black JD and Azizkhan-Clifford J: Sp1 and kruppellike factor family of transcription factors in cell growth regulation and cancer. J Cell Physiol 188: 143-160, 2001.
26. Li L, He S, Sun JM, et al: Gene regulation by Sp1 and Sp3. Biochem Cell Biol 82: 460-471, 2004.

27. Mukhopadhyay D and Datta K: Multiple regulatory pathways of vascular permeability factor/vascular endothelial growth factor (VPF/VEGF) expression in tumors. Semin Cancer Biol 14: 123-130, 2004.

28. Abdelrahim M, Smith R III, Burghardt R and Safe S: Role of Sp proteins in regulation of vascular endothelial growth factor expression and proliferation of pancreatic cancer cells. Cancer Res 64: 6740-6749, 2004

29. Safe $\mathrm{S}$ and Abdelrahim M: Sp transcription factor family and its role in cancer. Eur J Cancer 41: 2438-2448, 2005.

30. Wohlert SE, Kunzel E, Machinek R, et al: The structure of mithramycin reinvestigated. J Nat Prod 62: 119-121, 1999.

31. Prado L, Lombo F, Brana AF, et al: Analysis of two chromosomal regions adjacent to genes for a type II polyketide synthase involved in the biosynthesis of the antitumor polyketide mithramycin in Streptomyces argillaceus. Mol Gen Genet 261: 216-225, 1999.

32. Hall TJ, Schaeublin M and Chambers TJ: The majority of osteoclasts require mRNA and protein synthesis for bone resorption in vitro. Biochem Biophys Res Commun 195: 1245-1253, 1993.

33. Remsing LL, Bahadori HR, Carbone GM, et al: Inhibition of c-src transcription by mithramycin: structure-activity relationships of biosynthetically produced mithramycin analogues using the c-src promoter as target. Biochemistry 42: 8313-8324, 2003.

34. Chatterjee S, Zaman K, Ryu H, et al: Sequence-selective DNA binding drugs mithramycin $\mathrm{A}$ and chromomycin A3 are potent inhibitors of neuronal apoptosis induced by oxidative stress and DNA damage in cortical neurons. Ann Neurol 49: 345-354, 2001.

35. Blume SW, Snyder RC, Ray R, et al: Mithramycin inhibits SP1 binding and selectively inhibits transcriptional activity of the dihydrofolate reductase gene in vitro and in vivo. J Clin Invest 88: 1613-1621, 1991 .

36. Tagashira M, Kitagawa $\mathrm{T}$, Isonishi S, et al: Mithramycin represses MDR1 gene expression in vitro, modulating multidrug resistance. Biol Pharm Bull 23: 926-929, 2000.

37. Duverger V, Murphy AM, Sheehan D, et al: The anticancer drug mithramycin A sensitises tumour cells to apoptosis induced by tumour necrosis factor (TNF). Br J Cancer 90: 2025-2031, 2004.

38. Koutsodontis $\mathrm{G}$ and Kardassis D: Inhibition of p53-mediated transcriptional responses by mithramycin A. Oncogene 23: 9190-9200, 2004

39. Weidner N, Semple JP, Welch WR, et al: Tumor angiogenesis and metastasis-correlation in invasive breast carcinoma. $\mathrm{N}$ Engl J Med 324: 1-8, 1991.

40. Kanai M, Wei D, Li Q, et al: Kruppel-like factor 4 represses $\mathrm{Sp} 1$ expression and contributes to its tumor suppressor activity in human pancreatic and gastric cancer. Clin Cancer Res 12: 6395-6402, 2006.

41. Morabito A, De Maio E, Di Maio M, et al: Tyrosine kinase inhibitors of vascular endothelial growth factor receptors in clinical trials: current status and future directions. Oncologist 11: 753-764, 2006.

42. Yazici YD, Kim S, Jasser SA, et al: Antivascular therapy of oral tongue squamous cell carcinoma with PTK787. Laryngoscope 115: 2249-2255, 2005.

43. Prox D, Becker C, Pirie-Shepherd SR, et al: Treatment of human pancreatic cancer in mice with angiogenic inhibitors. World J Surg 27: 405-411, 2003.

44. Wiedmann MW and Caca K: Molecularly targeted therapy for gastrointestinal cancer. Curr Cancer Drug Targets 5: 171-193, 2005 .

45. Jain RK: Tumor angiogenesis and accessibility: role of vascular endothelial growth factor. Semin Oncol 29: 3-9, 2002.

46. Blagosklonny MV: How Avastin potentiates chemotherapeutic drugs: action and reaction in antiangiogenic therapy. Cancer Biol Ther 4: 1307-1310, 2005.

47. Kowanetz M and Ferrara N: Vascular endothelial growth factor signaling pathways: therapeutic perspective. Clin Cancer Res 12: 5018-5022, 2006. 